\title{
Benefits of blocking fructokinase
}

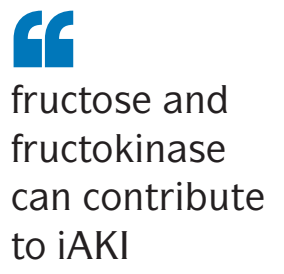

g
Fructose metabolism has been implicated in the progression of kidney disease. Now, Ana Andres-Hernando et al. show that renal production of endogenous fructose has a role in the pathogenesis of ischaemic acute kidney injury (iAKI) in mice, and that blocking fructokinase - which mediates the first step in fructose metabolism - can protect against renal injury.

The polyol pathway uses aldose reductase and sorbitol dehydrogenase to generate sorbitol and fructose from glucose. The researchers found that fructose levels were elevated in the urine of patients with $\mathrm{AKI}$, and that induction of $\mathrm{iAKI}$ in mice led to an increase in the levels of aldose reductase, sorbitol and fructose in the kidney cortex. Thus, they suggest that the polyol pathway is activated in the kidney cortex in AKI.

To determine the role of fructose in iAKI the researchers generated fructokinasedeficient mice. Following iAKI, these mice had less severe renal injury and lower levels of inflammation, serum creatinine and blood urea nitrogen (BUN) and displayed less severe renal injury than wild-type controls. In addition, fructokinase-deficient mice, but not wild-type mice, rapidly restored their renal ATP levels following iAKI. These findings suggest that fructokinase deficiency can protect against $\mathrm{iAKI}$. Interestingly, radiocontrast agents, which activate the polyol pathway and cause nephropathy, were also less detrimental to the kidney in fructokinase-deficient mice than in wild-type mice.

Finally, the researchers show that intravenous administration of luteolin - a naturally occurring flavone that can inhibit fructokinase - reduced levels of serum creatinine, BUN and markers of tubular injury in mice undergoing iAKI as compared to levels in mice treated with vehicle. Thus, they conclude that fructose and fructokinase can contribute to iAKI and could be potential therapeutic targets to prevent iAKI and/or accelerate renal recovery. Katharine H. Wrighton

ORIGINAL ARTICLE Andres-Hernando, A. et al. Protective role of fructokinase blockade in the pathogenesis of acute kidney injury in mice. Nat. Commun. http://dx.doi.org/10.1038/ ncomms14181 (2017) 